and colleagues (5) establishes a framework for how to tease out genetic and biochemical modifiers of complex phenotypes in monogenic disorders such as IBMPFD and neurofibromatosis type 1 .

\section{Acknowledgments}

Conrad C. Weihl is supported by the NIH (R01 AG031867) and the Muscular Dystrophy Association.

Address correspondence to: Conrad C. Weihl, Department of Neurology, Washington University School of Medicine, 660 S. Euclid Avenue, Saint Louis, Missouri 63110, USA. Phone: 314.362.6981; Fax: 314.362.4391; E-mail:weihlc@neuro.wustl.edu.

\footnotetext{
1. Watts GD, et al. Inclusion body myopathy associated with Paget disease of bone and frontotemporal dementia is caused by mutant valosin-containing protein. Nat Genet. 2004;36(4):377-381.

2. Weihl CC. Valosin containing protein associated fronto-temporal lobar degeneration: clinical presentation, pathologic features and pathogenesis. Curr Alzheimer Res. 2011;8(3):252-260.

3. Majounie E, et al. Mutational analysis of the VCP gene in Parkinson's disease [published online ahead of print September 13, 2011]. Neurobiol Aging. doi:10.1016/j.neurobiolaging.2011.07.011.
}

4. Johnson JO, et al. Exome sequencing reveals VCP mutations as a cause of familial ALS. Neuron. 2010;68(5):857-864.

5. Wang H-F, Shih Y-T, Chen C-Y, Chao H-W, Lee M-J, Hsueh Y-P. Valosin-containing protein and neurofibromin interact to regulate dendritic spine density. J Clin Invest. 2011;121(12):4820-4837.

6. Halawani D, Latterich M. p97: The cell's molecular purgatory? Mol Cell. 2006;22(6):713-717.

7. Ramadan K, et al. Cdc48/p97 promotes reformation of the nucleus by extracting the kinase Aurora B from chromatin. Nature. 2007;450(7173):1258-1262.

8. Rabinovich E, Kerem A, Frohlich KU, Diamant N, BarNun S. AAA-ATPase p97/Cdc48p, a cytosolic chaperone required for endoplasmic reticulum-associated protein degradation. Mol Cell Biol. 2002;22(2):626-634.

9. Dreveny I, et al. p97 and close encounters of every kind: a brief review. Biochem Soc Trans. 2004; 32(pt 5):715-720.

10. Ritz D, et al. Endolysosomal sorting of ubiquitylated caveolin-1 is regulated by VCP and UBXD1 and impaired by VCP disease mutations. Nat Cell Biol. 2011;13(9):1116-1123.

11. Fernandez-Saiz V, Buchberger A. Imbalances in $p 97$ co-factor interactions in human proteinopathy. EMBO Rep. 2010;11(6):479-485.

12. Ju JS, Miller SE, Hanson PI, Weihl CC. Impaired protein aggregate handling and clearance underlie the pathogenesis of $\mathrm{p} 97 / \mathrm{VCP}$-associated disease. J Biol Chem. 2008;283(44):30289-30299.

13. Ju JS, et al. Valosin-containing protein (VCP) is required for autophagy and is disrupted in VCP disease. J Cell Biol. 2009;187(6):875-888.

14. Tanaka A, et al. Proteasome and p97 mediate mitophagy and degradation of mitofusins induced by Parkin. J Cell Biol. 2010;191(7):1367-1380.

15. Weihl CC, Dalal S, Pestronk A, Hanson PI. Inclusion body myopathy-associated mutations in $\mathrm{p} 97 /$ VCP impair endoplasmic reticulum-associated degradation. Hum Mol Genet. 2006;15(2):189-199.

16. Janiesch PC, et al. The ubiquitin-selective chaperone CDC-48/p97 links myosin assembly to human myopathy. Nat Cell Biol. 2007;9(4):379-390.

17. Landsverk ML, et al. The UNC-45 chaperone mediates sarcomere assembly through myosin degradation in Caenorhabditis elegans. J Cell Biol. 2007;177(2):205-210.

18. Phan VT, Ding VW, Li F, Chalkley RJ, Burlingame A, McCormick F. The RasGAP proteins Ira2 and neurofibromin are negatively regulated by Gpb1 in yeast and ETEA in humans. Mol Cell Biol. 2010;30(9):2264-2279.

19. Sugita S, Sudhof TC. Specificity of Ca2+-dependent protein interactions mediated by the C2A domains of synaptotagmins. Biochemistry. 2000;39(11):2940-2949.

20. Clemen CS, et al. Strumpellin is a novel valosincontaining protein binding partner linking hereditary spastic paraplegia to protein aggregation diseases. Brain. 2010;133(10):2920-2941.

21. Valdmanis PN, et al. Mutations in the KIAA0196 gene at the SPG8 locus cause hereditary spastic paraplegia. Am J Hum Genet. 2007;80(1):152-161.

22. Rumpf S, Lee SB, Jan LY, Jan YN. Neuronal remodeling and apoptosis require VCP-dependent degradation of the apoptosis inhibitor DIAP1. Development. 2011;138(6):1153-1160.

23. Shilyansky C, Lee YS, Silva AJ. Molecular and cellular mechanisms of learning disabilities: a focus on NF1. Annu Rev Neurosci. 2010;33:221-243.

\title{
On the origin of the liver
}

\author{
Joshua R. Friedman ${ }^{1}$ and Klaus H. Kaestner ${ }^{2}$ \\ 1Department of Pediatrics, ${ }^{2}$ Department of Genetics, and Institute for Diabetes, Obesity, and Metabolism, \\ Perelman School of Medicine, University of Pennsylvania, Philadelphia, Pennsylvania, USA.
}

\begin{abstract}
While it has been well established that the fetal liver originates from foregut endoderm, the identity of the mechanisms that maintain liver mass under both basal and injury conditions remains controversial. Dramatically different models have been proposed based on the experimental design employed. In this issue of the JCI, Malato and colleagues report their elegant new model for genetic lineage tracing of mature mouse hepatocytes using an adenoassociated virus-driven Cre recombinase. They show convincingly that maintenance of liver mass during normal turnover or in response to mild injury is achieved by mature hepatocytes, rather than cholangiocytes or specialized progenitor cells, as has been suggested by others.
\end{abstract}

For parents, the question, where do babies come from? may cause dread or discomfort. The same is true for scientists who are asked, where do hepatocytes come from? The reason for this is that there is not a single answer. Hepatocytes can arise from different cell types depending on age (pre-

Conflict of interest: Klaus H. Kaestner consults for Johnson \& Johnson.

Citation for this article: J Clin Invest. 2011; 121(12):4630-4633. doi:10.1172/JCI59652. natal vs. adult), the presence or absence of hepatic injury, and even the type and degree of injury. An understanding of hepatocyte origins is a prerequisite for advancing the treatment of liver disease by promoting regeneration of endogenous hepatocytes or by deriving hepatocytes ex vivo for cellular therapy. The work of Malato and colleagues, reported in this issue of the JCI (1), adds new and important information regarding the origins of adult hepatocytes, although controversy still remains.

\section{Fetal hepatocytes are derived from hepatoblasts}

One area of clarity regarding hepatocyte origin is that embryonic and fetal hepatocytes arise from hepatoblasts, endoderm-derived cells with the potential to differentiate into either hepatocytes, which populate the bulk of the liver parenchyma, or cholangiocytes, which line the intrahepatic bile ducts. Cholangiocytes differentiate from hepatoblasts near a transient fetal structure called the ductal plate, while the remaining hepatoblasts differentiate into hepatocytes (2). Hepatoblasts are characterized by coexpression of biliary markers (e.g., cytokeratin 19) and hepatocyte markers (e.g., albumin) as well as hepatoblastspecific genes (e.g., $\alpha$-fetoprotein). Shortly after birth, this cell type is virtually undetectable in situ. This implies that the supply of hepatocytes necessary for the rapid hepatic growth that occurs in juveniles must arise from another cell type. 
A
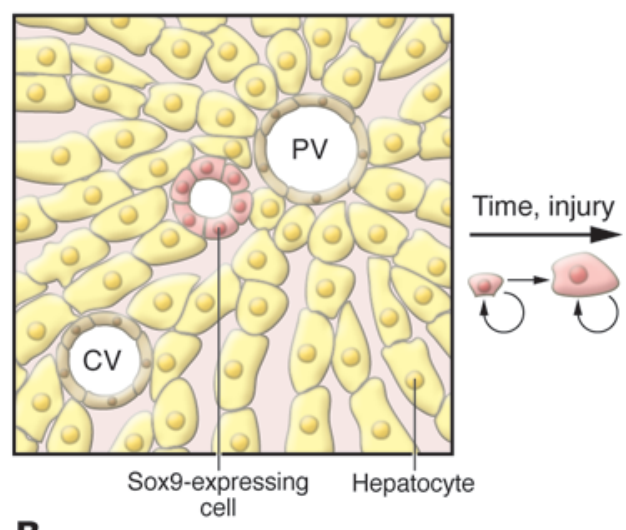

B
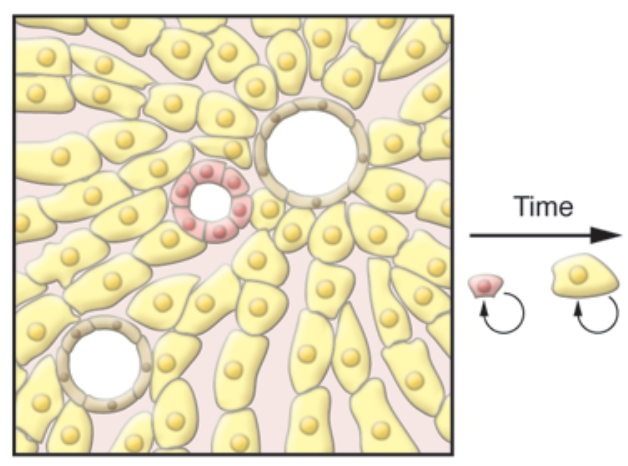

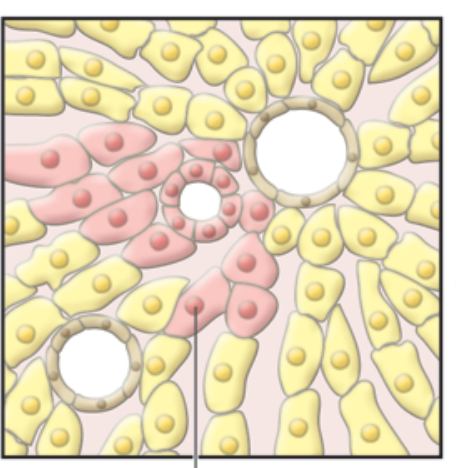

Hepatocyte derived from Sox9-expressing cell

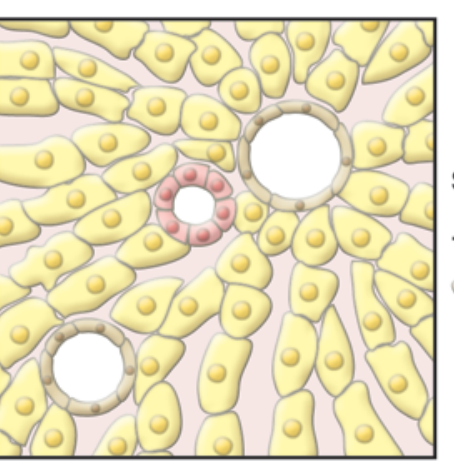

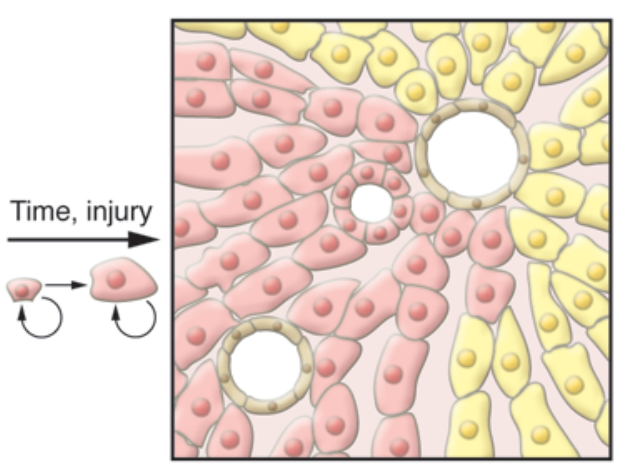

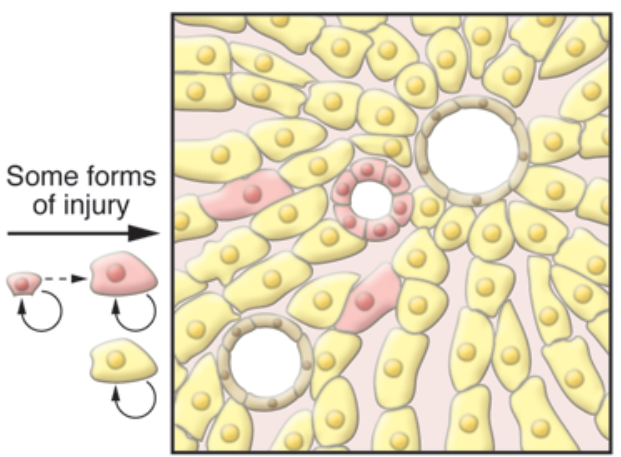

Figure 1

Comparison of 2 models of liver cell homeostasis using pulse labeling. In this hypothetical example, Sox9-expressing cells (pink) and hepatocytes (yellow) are permanently marked, as are all their progeny. (A) In the model proposed by Furuyama et al. (17), after labeling, Sox9-expressing cells differentiate into both cholangiocytes and hepatocytes, ultimately populating the entire lobule. This requires streaming of the Sox9-derived hepatocytes from zone 1 to zone 3. (B) The data presented by Malato and colleagues in this issue of the $\mathrm{JCl}$ (1) support a distinct model in which, under most conditions, hepatocytes are derived from other hepatocytes, without any contribution from nonhepatocytes. In some forms of injury, a small percentage of hepatocytes are derived from a nonhepatocyte cell population, perhaps from Sox9-expressing stem cells.

\section{Postnatal hepatocytes are derived from hepatocytes}

The earliest studies investigating adult hepatocyte origin were based on partial (two-thirds) hepatectomy in the rat. Seventy-four years ago (16 years before the identification of DNA as the genetic material), Brues and Marble noted (3) that after partial hepatectomy, "[m]itoses are evenly distributed throughout the liver and throughout each lobule; there is no preponderance near the bile duct cells.” They concluded that hepatocytes themselves are the predominant source of new hepatocytes, and this model has been supported by work in many laboratories (4). Indeed, studies using serial transplantation of hepatocytes have shown that small numbers of hepatocytes are capable of fully reconstituting diseased livers multiple times, dividing more than 100 times in the process (5).

Until recently, it was accepted that the same process of hepatocyte self renewal is responsible for homeostasis in all cases in which healthy hepatocytes are available in sufficient numbers, such as in the quiescent liver or following mild injury. In 1962, Grisham used tritiated thymidine to pulse-label replicating DNA following partial hepatectomy in rats (6) and observed that the first hepatocytes to be labeled were those closest to the portal tracts, regions of the liver known as zones 1 and 2. Labeled zone 3 hepatocytes were seen nearly a day later. This finding led to a model in which new hepatocytes arise in the periportal area, after which they "stream" out to populate the remainder of the lobule. While the "streaming" hypothesis gained some support $(7,8)$, other data suggested that streaming of hepatocytes does not occur (9, 10). Mice harboring a transgene expressing lac $Z$ under the control of the hepatocytespecific $\alpha$-1-antitrypsin promoter have mosaic expression of lac $Z$ in hepatocytes (9). On its own, this is uninformative, but observations in transgenic mice of different ages (or after injury) demonstrated that with time, the clusters of lacZ-positive cells grew larger and the overall distribution of the clusters did not change. This is incompatible with the streaming hypothesis, which predicts that later clones of lacZpositive cells will be observed closer to the central vein. Identical results were obtained in experiments in which retroviral infection (which requires replicating cells) was used to mark rat hepatocytes one day after partial hepatectomy (10). Thus, these studies $(9,10)$ strongly suggest that streaming does not occur in the quiescent liver or following partial hepatectomy.

As Grisham himself pointed out (11), the appearance of streaming, seen in both his own and others' experiments, may be an artifact of the use of radiolabeled thymidine, in which the initial pulse of labeled thymidine is released from cell populations that are rapidly turning over. This would permit other cells - such as zone 3 hepatocytes - to become labeled after the pulse phase is completed. 


\section{Hepatocytes are derived from progenitor cells when the supply of healthy hepatocytes is insufficient}

While hepatocytes are a self-renewing population under normal conditions, a variety of experimental models have demonstrated that a nonhepatocyte population can also supply new hepatocytes. A common feature of all the models in which this has been demonstrated is that hepatocyte self renewal is prevented, either by an exogenous toxin or as a result of cholestatic injury (4). In this case, a progenitor cell population (known as oval cells in rodents) appears to give rise to both hepatocytes and cholangiocytes (4). Determining the precise identity of these progenitor cell populations has been challenging, most likely because they may not comprise a single cell type and because several of the markers used are not specific to progenitors. Nevertheless, it is clear that progenitors arise from cells in or near the canals of Hering (the intrahepatic bile ductules in direct communication with the hepatocyte canalicular system), are likely a component of the periportal proliferation of cholangiocytes seen in response to injury (the so-called ductular reaction), and are able to repopulate injured liver, even from single-cell clones $(12,13)$. At least some of these cells are identified by expression of the forkhead box transcription factor FoxL1 (14). Finally, while progenitor cells residing outside the liver may be capable of differentiating into hepatocyte-like cells $(15,16)$, they are not likely to be relevant under most conditions.

\section{Sox9-expressing cells as hepatocyte progenitors: new life for the streaming hypothesis?}

In late 2010, Furuyama and colleagues published a remarkable paper in which they studied liver cells expressing the highmobility group transcription factor Sox 9 (17). Sox9 is expressed in the ductal plate and is required for the proper timing of fetal cholangiocyte differentiation (18); it is also required for the maintenance of pancreatic progenitor cells (19). To investigate the possibility that Sox9 is also expressed in hepatic progenitor cells, Furuyama and colleagues used homologous recombination in ES cells to place a CreERT2-encoding gene into the Sox9 locus (17). CreERT2 is a fusion protein of the Cre recombinase and a mutated estrogen receptor; it is able to recombine loxP sites only in the presence of tamoxifen. This tool allowed the investigators to perform pulse labeling similar to that in
Grisham's 1962 study (6), but marking only Sox9-expressing cells. At early time points, the marked cells were found to be present in bile ducts, as would be expected based on Sox9 immunohistochemistry (Figure 1A).

However, the appearance of marked cells in the liver at later time points was unexpected: over the course of one year, the marked cells assumed the morphology of hepatocytes and expanded toward the central vein, ultimately representing a majority of the hepatocyte population (Figure 1A) (17). This process was accelerated in the $\mathrm{CCl}_{4}$ and bile duct ligation models of hepatic injury, indicating that Sox9-expressing cells are also hepatocyte progenitors in the context of these forms of hepatic injury. However, Sox9-expressing cells did not contribute significantly to regeneration following partial hepatectomy or in other injury models.

This ability of cholangiocyte-like Sox $9^{+}$ cells to function as hepatocyte progenitors after injury is consistent with prior studies of oval cells (which resemble cholangiocytes) and other hepatocyte progenitors that are also located in the portal area, although it remains to be demonstrated that the Sox $9^{+}$cells share markers with oval cells. The surprising aspect of the study by Furuyama and colleagues was their finding that the full population of adult hepatocytes was derived from Sox9 $9^{+}$cells that were cholangiocytes in initial morphology (17). This model supports a return to the streaming hypothesis, in apparent contradiction with the aforementioned lineage-tracing experiments $(9,10)$.

\section{A new method of pulse labeling hepatocytes for lineage tracing}

In this issue of the JCI, Malato and colleagues use a novel technique to lineage trace pulse-labeled hepatocytes (1). Others have used tamoxifen-regulated Cre recombinase to mark hepatocytes (e.g., ref. 17), and there are concerns that the promoters used result in incomplete or nonspecific labeling of cells and that the toxicity of tamoxifen can affect experimental outcomes. To overcome these issues, Malato et al. used a novel vector based on adenoassociated virus serotype 8 (AAV8), which exhibits tropism for hepatocytes. The AAV8-Ttr-Cre vector designed by Malato and colleagues drives expression of Cre recombinase under the control of the hepatocyte-specific transthyretin (Ttr) promoter, adding a level of hepatocyte specificity to that imposed by the use of the AAV8-based vector. The vec- tor did not integrate into the host genome and did not replicate or reinfect other cells, so the treatment of ROSA26-EYFP (where EYFP indicates enhanced yellow fluorescent protein) mice with AAV8-Ttr-Cre was equivalent to a pulse labeling of hepatocytes. This approach is therefore the complement of the cholangiocyte labeling performed by Furuyama and colleagues (17).

Malato and colleagues first confirmed that treatment with AAV8-Ttr-Cre resulted in EYFP expression in all hepatocytes (1). Importantly, this was achieved without any serum biochemical evidence of hepatotoxicity. They also confirmed that no Sox $9^{+}$cells were marked. The model proposed by Furuyama and colleagues (17) predicts that over time, the percentage of $\mathrm{EYFP}^{+}$hepatocytes should decrease, as they are replaced by daughters of the Sox $9^{+}$/ EYFP $^{-}$cholangiocyte-like cells. However, at 12 weeks after infection, Malato and colleagues could detect no EYFP- hepatocytes (Figure 1B) (1). This observation is in direct contrast with the finding of Furuyama and colleagues that many hepatocytes were derived from Sox $9^{+}$cells 2 months after tamoxifen treatment (17).

Malato and colleagues also show that induction of liver regeneration as a result of acute $\mathrm{CCl}_{4}$ toxicity resulted in no $\mathrm{EYFP}^{-}$ hepatocytes at two days after treatment (Figure 1B), when the peak of replication is expected to occur (1). A few EYFPhepatocytes were observed after 6 weeks of chronic low $\mathrm{CCl}_{4}$ exposure, but at a much lower frequency than expected based on the results of Furuyama and colleagues (17). The induction of liver regeneration by partial hepatectomy also resulted in an extremely low frequency of unmarked hepatocytes (1); this is consistent with the lack of hepatocytes derived from Sox $9^{+}$ cells in the same injury model. No EYFPhepatocytes were observed after bile duct ligation or 3,5-diethoxycarbonyl-1,4-dihydrocollidine (DDC) feeding; only the latter case is in agreement with the findings of Furuyama and colleagues (17). Both of these models of hepatic injury induced proliferation of Sox9+ bile duct cells, but none of these cells gave rise to hepatocytes.

\section{Conclusions}

How can these disparate results of Malato and colleagues (1) and Furuyama and colleagues (17) be reconciled? The most striking difference between the two studies is in the case of hepatocyte homeostasis in the quiescent liver; in the injury models, 
it is likely that both preexisting mature hepatocytes and Sox $9^{+}$cells contribute to regeneration. One possibility is that the "quiescent" liver in the Sox9 knockin mice (17) is actually undergoing chronic injury, either via decreased Sox9 levels or toxicity due to tamoxifen or chronic CreERT2 expression. However, this would have to cause a non-cell autonomous inhibition of hepatocyte replication to result in the sort of oval/progenitor cell-derived repopulation of the liver seen in the DDC diet. By using a conditional lineage-tracing model that does not produce any measurable toxicity or liver injury, Malato and colleagues (1) provide strong evidence that it is hepatocytes themselves, and not cholangiocytes or specialized progenitor cells, that are responsible for the homeostatic renewal of the liver under basal and even mild injury conditions.

\section{Acknowledgments}

Related work in K.H. Kaestner's lab is supported by P01 DK049210 and in J.R. Friedman's lab by R01 DK079881. We thank our colleagues in the liver field for many stimulating discussions on the subject.
Address correspondence to: Klaus $\mathrm{H}$. Kaestner, University of Pennsylvania, 560A CRB/6140, Philadelphia, Pennsylvania, USA. Phone: 215.898.8759; Fax: 215.573.5892; E-mail: kaestner@mail.med.upenn.edu.

1. Malato Y, et al. Fate tracing of mature hepatocytes in mouse liver homeostasis and regeneration. JClin Invest. 2011;121(12):4850-4860.

2. Zorn AM. Liver development. In: Girard L, ed. StemBook. Cambridge, Massachusetts, USA: Harvard Stem Cell Institute; 2008.

3. Brues AM, Marble BB. An analysis of mitosis in liver restoration. J Exp Med. 1937;65(1):15-27.

4. Duncan AW, Dorrell C, Grompe M. Stem cells and liver regeneration. Gastroenterology. 2009;137(2):466-481.

5. Overturf K, al-Dhalimy M, Ou CN, Finegold M, Grompe M. Serial transplantation reveals the stem-cell-like regenerative potential of adult mouse hepatocytes. Am J Path. 1997;151(5):1273-1280.

6. Grisham JW. A morphologic study of deoxyribonucleic acid synthesis and cell proliferation in regenerating rat liver; autoradiography with thymidineH3. Cancer Res. 1962;22:842-849.

7. Zajicek G, Oren R, Weinreb M Jr. The streaming liver. Liver. 1985;5(6):293-300

8. Fletcher J, et al. The inhibitory role of stromal cell mesenchyme on human embryonic stem cell hepatocyte differentiation is overcome by Wnt3a treatment. Cloning Stem Cells. 2008; 10(3):331-339.

9. Kennedy S, Rettinger S, Flye MW, Ponder KP. Experiments in transgenic mice show that hepatocytes are the source for postnatal liver growth and do not stream. Hepatology. 1995;22(1):160-168.

10. Bralet MP, Branchereau S, Brechot C, Ferry N. Cell lineage study in the liver using retroviral mediated gene transfer. Evidence against the streaming of hepatocytes in normal liver. Am J Pathol. 1994;144(5):896-905.

11. Grisham JW. Grisham: reply. Am J Pathol. 1995; 146(3):773-776.

12. Suzuki A, et al. Flow cytometric isolation and clonal identification of self-renewing bipotent hepatic progenitor cells in adult mouse liver. Hepatology. 2008;48(6):1964-1978.

13. Wang X, Foster M, Al-Dhalimy M, Lagasse E, Finegold M, Grompe M. The origin and liver repopulating capacity of murine oval cells. Proc Natl Acad Sci US A. 2003;100 suppl 1:11881-11888.

14. Shin S, et al. Foxl1-Cre-marked adult hepatic progenitors have clonogenic and bilineage differentiation potential. Genes Dev. 2011;25(11):1185-1192.

15. Schwartz RE, et al. Multipotent adult progenitor cells from bone marrow differentiate into functional hepatocyte-like cells. J Clin Invest. 2002;109(10):1291-1302.

16. Jiang $\mathrm{Y}$, et al. Pluripotency of mesenchymal stem cells derived from adult marrow. Nature. 2002; 418(6893):41-49.

17. Furuyama K, et al. Continuous cell supply from a Sox9-expressing progenitor zone in adult liver, exocrine pancreas and intestine. Nat Genet. 2011; 43(1):34-41.

18. Antoniou A, et al. Intrahepatic bile ducts develop according to a new mode of tubulogenesis regulated by the transcription factor SOX9. Gastroenterology. 2009;136(7):2325-2333.

19. Seymour PA, et al. SOX9 is required for maintenance of the pancreatic progenitor cell pool. Proc Natl Acad Sci U S A. 2007;104(6):1865-1870.

\title{
Perception of sound and gravity by TMC1 and TMC2
}

Xi Lin

Departments of Otolaryngology and Cell Biology, Emory University School of Medicine, Atlanta, Georgia, USA.

\begin{abstract}
Central to our ability to hear and sense gravity is a cellular process known as mechanotransduction, which is initiated by the opening of mechanosensitive cation channels located near the tips of the stereocilia of auditory and vestibular inner ear hair cells. The molecular identity of the mechanotransduction channels has eluded researchers despite intensive investigations over the years. In this issue of the JCI, Kawashima et al. report their results obtained using mice with targeted deletion of both transmembrane channel-like 1 (Tmc1) and $T m c 2$. The use of inner ear hair cells isolated from these mice provided a nearly perfect system for testing the mechanotransduction channels without disrupting functions of other accessory proteins needed in the complicated molecular apparatus, and it allowed the authors to show that the proteins encoded by these genes are integral components of the mechanotransduction complex.
\end{abstract}

Auditory and vestibular inner ear hair cells are central to our ability to perceive sound

Conflict of interest: The author has declared that no conflict of interest exists.

Citation for this article: J Clin Invest. 2011; 121(12):4633-4636. doi:10.1172/JCI61167. and gravity, respectively. These cells convert mechanical stimuli initiated by sounds and gravitational force into electrical signals, a process known as mechanotransduction. Mechanotransduction is initiated when the mechanical stimuli cause stereocilia at the apical surface of the hair cells to move, triggering the opening of mechanosensitive cation channels located at the tip. Ionic currents that flow through the open channels initiate graded receptor potentials (refs. 1, 2, and Figure 1). This results in rapid changes in the membrane potential of the hair cells and subsequently modulates neurotransmitter release at the hair cell base. Auditory and vestibular inner ear hair cells synapse with auditory and vestibular nerves, respectively. Thus, changes in neurotransmitter release by the hair cells modulate postsynaptic action potentials to convey the mechanical information to the brain. These neuronal activities underlie our perception of sound and are necessary for maintaining balance and coordination of eye and head movements.

Molecular identification of the mechanotransduction channels in the stereocilia 